\title{
On fixed point theorems for mappings with PPF dependence
}

\author{
Ali Farajzadeh ${ }^{1}$ and Anchalee Kaewcharoen ${ }^{2,3^{*}}$
}

"Correspondence:

anchaleeka@nu.ac.th

${ }^{2}$ Department of Mathematics,

Faculty of Science, Naresuan

University, Phitsanulok, 65000,

Thailand

${ }^{3}$ Centre of Excellence in

Mathematics, CHE, Si Ayutthaya Rd.

Bangkok, 10400, Thailand

Full list of author information is

available at the end of the article

\begin{abstract}
In this paper, we consider two families $\Psi_{1}$ and $\Psi_{2}$ of mappings defined on $[0,+\infty)$ satisfy some certain properties. Using the mentioned properties for $\Psi_{1}$ and $\Psi_{2}$, we prove the analogous PPF dependent fixed point theorems for mappings as in Drici et al. (Nonlinear Anal. 67:641-647, 2007) in partially ordered Banach spaces where mappings satisfy the weaker contractive conditions without assuming the topological closedness with respect to the norm topology for the Razumikhin class $\mathcal{R}_{c}$.
\end{abstract}

MSC: $54 \mathrm{H} 25 ; 55 \mathrm{M} 20$

Keywords: PPF dependent fixed points; Razumikhin classes; partially ordered sets; algebraic closedness with respect to difference

\section{Introduction and preliminary results}

The fixed point theorems for mappings satisfying certain contractive conditions have been continually studied for decade (see [1-9] and references contained therein). Bernfeld et al. [10] proved the existence of PPF (past, present and future) dependent fixed points in the Razumikhin class for mappings that have different domains and ranges. After that Dhage [11] extended the existence of PPF dependent fixed points to PPF common dependent fixed points for mappings satisfying the weaker contractive conditions. In 2007, Drici et al. [2] proved the fixed point theorems in partially ordered metric spaces for mappings with PPF dependence. In this paper, we consider two families $\Psi_{1}$ and $\Psi_{2}$ of mappings defined on $[0,+\infty)$ satisfy some certain properties. Moreover, the PPF dependent fixed point theorems for mappings satisfying some generalized contractive conditions in partially ordered Banach spaces are proven using the mentioned properties for $\Psi_{1}$ and $\Psi_{2}$.

Suppose that $E$ is a real Banach space with the norm $\|\cdot\|_{E}$ and $I$ is a closed interval $[a, b]$ in $\mathbb{R}$. Let $E_{0}=C(I, E)$ be the set of all continuous $E$-valued mappings on $I$ equipped with the supremum norm $\|\cdot\|_{E_{0}}$ defined by

$$
\|\phi\|_{E_{0}}=\sup _{t \in I}\|\phi(t)\|_{E^{\prime}}
$$

for all $\phi \in E_{0}$. For a fixed element $c \in I$, the Razumikhin class of mappings in $E_{0}$ is defined by

$$
\mathcal{R}_{c}=\left\{\phi \in E_{0}:\|\phi\|_{E_{0}}=\|\phi(c)\|_{E}\right\} .
$$

○2014 Farajzadeh and Kaewcharoen; licensee Springer. This is an Open Access article distributed under the terms of the Creative Commons Attribution License (http://creativecommons.org/licenses/by/2.0), which permits unrestricted use, distribution, and reproduction in any medium, provided the original work is properly cited. 
Recall that a point $\phi \in E_{0}$ is said to be a PPF dependent fixed point or a fixed point with PPF dependence of $T: E_{0} \rightarrow E$ if $T \phi=\phi(c)$ for some $c \in I$.

Example 1.1 Let $T: C([0,1], \mathbb{R}) \rightarrow \mathbb{R}$ be defined by

$$
T \phi=\frac{1}{2}\left(\sup _{t \in[0,1]}|\phi(t)|\right) \quad \text { for all } \phi \in C([0,1], \mathbb{R})
$$

Therefore $T$ is a contraction with a constant $\frac{1}{2}$. Suppose that $\phi(t)=t^{2}+1$ for all $t \in[0,1]$. Since $T \phi=\frac{1}{2}\left(\sup _{t \in[0,1]}|\phi(t)|\right)=1=\phi(0)$, we find that $\phi$ is a fixed point with dependence of $T$.

Definition 1.2 Let $A$ be a subset of $E$. Then

(i) $A$ is said to be topologically closed with respect to the norm topology if for each sequence $\left\{x_{n}\right\}$ in $A$ with $x_{n} \rightarrow x$ as $n \rightarrow \infty$ implies $x \in A$.

(ii) $A$ is said to be algebraically closed with respect to the difference if $x-y \in A$ for all $x, y \in A$.

Recently, Dhage [11] proved the existence of PPF fixed points for mappings satisfying the condition of Cirić type generalized contraction assuming topological closedness with respect to the norm topology for a Razumikhin class.

Definition 1.3 (Dhage, [11]) A mapping $T: E_{0} \rightarrow E$ is said to satisfy the condition of Cirić type generalized contraction if there exists a real number $\lambda \in[0,1)$ satisfying

$$
\begin{aligned}
\|T \phi-T \alpha\| \leq & \lambda \max \left\{\|\phi-\alpha\|_{E_{0}},\|\phi(c)-T \phi\|_{E},\|\alpha(c)-T \alpha\|_{E},\right. \\
& \left.\frac{1}{2}\left[\|\phi(c)-T \alpha\|_{E}+\|\alpha(c)-T \phi\|_{E}\right]\right\}
\end{aligned}
$$

for all $\phi, \alpha \in E_{0}$ and for some $c \in I$.

Theorem 1.4 (Dhage, [11]) Suppose that $T: E_{0} \rightarrow E$ satisfies the condition of Ciric type generalized contraction. Assume that $\mathcal{R}_{c}$ is topologically closed with respect to the norm topology and is algebraically closed with respect to the difference, then $T$ has a unique PPF dependent fixed point in $\mathcal{R}_{c}$.

It is a natural question that the result of the previous theorem is still valid by omitting the topological closedness of $\mathcal{R}_{c}$. In the next result, we will answer the question.

Proposition 1.5 The Razumikhin class $\mathcal{R}_{c}$ is topologically closed with respect to the norm topology.

Proof Let $\left\{\phi_{n}\right\}$ be a sequence in $\mathcal{R}_{c}$ converging to $\phi$. This implies that

$$
\lim _{n \rightarrow \infty}\left\|\phi_{n}-\phi\right\|_{E_{0}}=0 \quad \text { where }\left\|\phi_{n}-\phi\right\|_{E_{0}}=\sup _{t \in I}\left\|\phi_{n}(t)-\phi(t)\right\|_{E^{*}}
$$


Therefore

$$
\lim _{n \rightarrow \infty}\left\|\phi_{n}\right\|_{E_{0}}=\|\phi\|_{E_{0}} \quad \text { and } \quad \lim _{n \rightarrow \infty}\left\|\phi_{n}(t)\right\|_{E}=\|\phi(t)\|_{E} \quad \text { for all } t \in I .
$$

Since $\phi_{n} \in \mathcal{R}_{c}$ for all $n \in \mathbb{N}$, we obtain $\left\|\phi_{n}\right\|_{E_{0}}=\left\|\phi_{n}(c)\right\|_{E}$. Therefore

$$
\lim _{n \rightarrow \infty}\left\|\phi_{n}(c)\right\|_{E}=\|\phi\|_{E_{0}}
$$

By the uniqueness of the limit, we have $\|\phi\|_{E_{0}}=\|\phi(c)\|_{E}$. Hence $\phi \in \mathcal{R}_{c}$ and thus $\mathcal{R}_{c}$ is topologically closed with respect to the norm topology.

Hence, using Proposition 1.5, we can drop the topological closedness with respect to the norm topology for $\mathcal{R}_{c}$ in Theorem 1.4.

The following example shows that the algebraic closedness with respect to the difference of Razumikhin class $\mathcal{R}_{c}$ may fail.

Example 1.6 Let $E_{0}=C([0,1], \mathbb{R})$ and $c=1$. If we take $\phi(t)=t^{2}$ and $\alpha(t)=t$ for all $t \in[0,1]$, then $\phi, \alpha \in \mathcal{R}_{c}$ while $\phi-\alpha \notin \mathcal{R}_{c}$.

Proposition 1.7 If the Razumikhin class $\mathcal{R}_{c}$ is algebraically closed with respect to the difference, then $\mathcal{R}_{c}$ is a convex set.

Proof Since $\mathcal{R}_{c}$ is algebraically closed with respect to the difference, we have $\mathcal{R}_{c}-\mathcal{R}_{c} \subseteq \mathcal{R}_{c}$. Using the fact that $-\mathcal{R}_{c}=\mathcal{R}_{c}$, we obtain $\mathcal{R}_{c}+\mathcal{R}_{c} \subseteq \mathcal{R}_{c}$. Since $\lambda \mathcal{R}_{c} \subseteq \mathcal{R}_{c}$ for all $\lambda \in[0,1]$, we get $\lambda \mathcal{R}_{c}+(1-\lambda) \mathcal{R}_{c} \subseteq \mathcal{R}_{c}$. Hence $\mathcal{R}_{c}$ is a convex set.

One can verify that Razumikhin class $\mathcal{R}_{c}$ is a cone (i.e., $\lambda \phi \in \mathcal{R}_{c}$, for each $\phi \in \mathcal{R}_{c}$ and $\lambda \geq 0$ ). Then by applying the previous theorem Razumikhin class $\mathcal{R}_{c}$ is a convex cone (also closed).

In 2007, Drici et al. [2] proved the following the fixed point theorems in partially ordered complete metric spaces for mappings with PPF dependence.

Theorem $1.8([2])$ Let $(E, d, \leq)$ be a partially ordered complete metric space and $T: E_{0} \rightarrow$ $E$ where $E_{0}=C(I, E)$ and $I=[a, b]$. Assume that

(i) $T$ is a nondecreasing mapping;

(ii) for all $\phi, \alpha \in E_{0}$ with $\phi \leq \alpha, d(T \phi, T \alpha) \leq k d_{0}(\phi, \alpha)$ where $d_{0}(\phi, \alpha)=\max _{s \in I} d(\phi(s), \alpha(s))$ and $k \in[0,1)$;

(iii) there exists a lower solution $\phi_{0}$ such that $\phi_{0}(c) \leq T \phi_{0}$;

(iv) $T$ is a continuous mapping or if $\left\{\phi_{n}\right\}$ is a nondecreasing sequence in $E_{0}$ converging to $\phi \in E_{0}$, then $\phi_{n} \leq \phi$ for all $n \in \mathbb{N}$.

Then $T$ has a PPF dependent fixed point in $E_{0}$.

It is a natural question if one can obtain the result of the aforementioned theorem for a generalized contraction (that is, condition (ii)). One of the aims of this paper is to answer the question by considering a general case of Ciric type generalized contractions.

In this paper, we consider two families $\Psi_{1}$ and $\Psi_{2}$ of mappings defined on $[0,+\infty)$ satisfying some certain properties. Using the mentioned properties for $\Psi_{1}$ and $\Psi_{2}$, we prove the 
analogous PPF dependent fixed point theorems for mappings as in [2] in partially ordered real Banach spaces where mappings satisfy the weaker contractive conditions.

\section{PPF dependent fixed points in partially ordered Banach spaces}

We begin this part with the consideration of the example of a partially ordered real Banach space. Recall that the set $B(X, \mathbb{R})$ of all bounded linear operators from a normed space $X$ into $\mathbb{R}$ is a real Banach space with a norm defined by

$$
\|f\|=\sup _{x \in X,\|x\|=1}|f(x)| \quad \text { for all } f \in B(X, \mathbb{R}) .
$$

We know that $B(X, \mathbb{R})$ is a partially ordered real Banach space with a partial order defined as follows:

$$
f \leq g \text { if and only if } f(x) \leq g(x) \text { for all } x \in X .
$$

From now on, let $(E, \leq)$ be a partially ordered real Banach space. In this paper, we use the following notations:

$$
\begin{aligned}
\Psi_{1}= & \{\psi: \psi:[0,+\infty) \rightarrow[0,+\infty) \text { is nondecreasing with } \\
& \left.\sum_{n=1}^{\infty} \psi^{n}(t)<\infty \text { for all } t \in(0,+\infty)\right\} \text { and } \\
\Psi_{2}= & \{\psi: \psi:[0,+\infty) \rightarrow[0,+\infty) \text { is continuous nondecreasing with } \\
& \psi(t)<t \text { for all } t \in(0,+\infty)\} .
\end{aligned}
$$

Lemma 2.1 ([12]) Suppose that $\psi:[0,+\infty) \rightarrow[0,+\infty)$. If $\psi$ is nondecreasing, then for each $t \in(0,+\infty), \lim _{n \rightarrow \infty} \psi^{n}(t)=0$ implies $\psi(t)<t$.

Hence the difference between an element of $\Psi_{1}$ and an element of $\Psi_{2}$ is continuity.

\section{Remark 2.2}

(i) It is easily seen that if $\psi:[0,+\infty) \rightarrow[0,+\infty)$ is nondecreasing and $\psi(t)<t$ for all $t \in(0,+\infty)$, then $\psi(0)=0$.

(ii) We can see that if $\psi:[0,+\infty) \rightarrow[0,+\infty), \psi(t)<t$ for all $t \in(0,+\infty)$ and $\psi(0)=0$, then $\psi$ is continuous at 0 .

We now prove the PPF dependent fixed point theorems for mappings satisfying the generalized contractive conditions concerning with $\psi \in \Psi_{1}$ without assuming the topological closedness with respect to the norm topology for the Razumikhin class $\mathcal{R}_{c}$.

Theorem 2.3 Suppose that $\psi \in \Psi_{1}, c \in I$ and $T: E_{0} \rightarrow$ Esatisfies the following conditions:

(i) $T$ is a nondecreasing mapping; 
(ii) for all $\phi, \alpha \in E_{0}$ with $\phi \leq \alpha$, we have

$$
\begin{aligned}
\|T \phi-T \alpha\|_{E} \leq & \psi\left(\operatorname { m a x } \left\{\|\phi-\alpha\|_{E_{0}},\|\phi(c)-T \phi\|_{E},\|\alpha(c)-T \alpha\|_{E},\right.\right. \\
& \left.\left.\frac{1}{2}\left[\|\phi(c)-T \alpha\|_{E}+\|\alpha(c)-T \phi\|_{E}\right]\right\}\right) ;
\end{aligned}
$$

(iii) there exists a lower solution $\phi_{0} \in \mathcal{R}_{c}$ such that $\phi_{0}(c) \leq T \phi_{0}$;

(iv) $T$ is a continuous mapping.

Assume that $\mathcal{R}_{c}$ is algebraically closed with respect to the difference. Then $T$ has a PPF dependent fixed point in $\mathcal{R}_{c}$.

Proof Since $\phi_{0} \in \mathcal{R}_{c}$ and $T \phi_{0} \in E$, there exists $x_{1} \in E$ such that $T \phi_{0}=x_{1}$. Choose $\phi_{1} \in \mathcal{R}_{c}$ such that $x_{1}=\phi_{1}(c)$. Since $\phi_{0}$ is a lower solution in $\mathcal{R}_{c}$ such that $\phi_{0}(c) \leq T \phi_{0}$, it follows that $\phi_{0} \leq \phi_{1}$. Using the algebraic closedness with respect to the difference of $\mathcal{R}_{c}$, this yields

$$
\left\|\phi_{0}-\phi_{1}\right\|_{E_{0}}=\left\|\phi_{0}(c)-\phi_{1}(c)\right\|_{E}
$$

By the fact that $T$ is nondecreasing, we obtain

$$
\phi_{1}(c)=T \phi_{0} \leq T \phi_{1}
$$

By induction, we can construct the sequence $\left\{\phi_{n}\right\}$ such that

$$
\begin{aligned}
& T \phi_{0}=\phi_{1}(c) \leq T \phi_{1}=\phi_{2}(c) \leq \cdots \leq T \phi_{n}=\phi_{n+1}(c) \leq T \phi_{n+1} \cdots \\
& \phi_{n} \leq \phi_{n+1} \quad \text { and } \quad\left\|\phi_{n}-\phi_{n+1}\right\|_{E_{0}}=\left\|\phi_{n}(c)-\phi_{n+1}(c)\right\|_{E^{\prime}}
\end{aligned}
$$

for all $n \in \mathbb{N} \cup\{0\}$. Assume that $\phi_{n-1}=\phi_{n}$ for some $n \in \mathbb{N}$. It follows that $\phi_{n-1}(c)=\phi_{n}(c)=$ $T \phi_{n-1}$. Therefore $T$ has a fixed point in $\mathcal{R}_{c}$. Suppose that $\phi_{n-1} \neq \phi_{n}$ for all $n \in \mathbb{N}$. Therefore, for each $n \in \mathbb{N}$, we obtain

$$
\begin{aligned}
\left\|\phi_{n}-\phi_{n+1}\right\|_{E_{0}}= & \left\|\phi_{n}(c)-\phi_{n+1}(c)\right\|_{E} \\
= & \left\|T \phi_{n}-T \phi_{n-1}\right\|_{E} \\
\leq & \psi\left(\operatorname { m a x } \left\{\left\|\phi_{n}-\phi_{n-1}\right\|_{E_{0}},\left\|\phi_{n}(c)-T \phi_{n}\right\|_{E},\left\|\phi_{n-1}(c)-T \phi_{n-1}\right\|_{E},\right.\right. \\
& \left.\left.\frac{1}{2}\left[\left\|\phi_{n}(c)-T \phi_{n-1}\right\|_{E}+\left\|\phi_{n-1}(c)-T \phi_{n}\right\|_{E}\right]\right\}\right) \\
= & \psi\left(\operatorname { m a x } \left\{\left\|\phi_{n}-\phi_{n-1}\right\|_{E_{0}},\left\|\phi_{n}(c)-\phi_{n+1}(c)\right\|_{E},\left\|\phi_{n-1}(c)-\phi_{n}(c)\right\|_{E},\right.\right. \\
& \left.\left.\frac{1}{2}\left[\left\|\phi_{n}(c)-\phi_{n}(c)\right\|_{E}+\left\|\phi_{n-1}(c)-\phi_{n+1}(c)\right\|_{E}\right]\right\}\right) \\
\leq & \psi\left(\max \left\{\left\|\phi_{n}-\phi_{n-1}\right\|_{E_{0}},\left\|\phi_{n}-\phi_{n+1}\right\|_{E_{0}}, \frac{1}{2}\left\|\phi_{n-1}-\phi_{n+1}\right\|_{E_{0}}\right\}\right) \\
\leq & \psi\left(\operatorname { m a x } \left\{\left\|\phi_{n}-\phi_{n-1}\right\|_{E_{0}},\left\|\phi_{n}-\phi_{n+1}\right\|_{E_{0}},\right.\right.
\end{aligned}
$$




$$
\begin{aligned}
& \left.\left.\frac{1}{2}\left\|\phi_{n-1}-\phi_{n}\right\|_{E_{0}}+\left\|\phi_{n}-\phi_{n+1}\right\|_{E_{0}}\right\}\right) \\
\leq & \psi\left(\max \left\{\left\|\phi_{n}-\phi_{n-1}\right\|_{E_{0}},\left\|\phi_{n}-\phi_{n+1}\right\|_{E_{0}}\right\}\right) .
\end{aligned}
$$

If $\max \left\{\left\|\phi_{n}-\phi_{n-1}\right\|_{E_{0}},\left\|\phi_{n}-\phi_{n+1}\right\|_{E_{0}}\right\}=\left\|\phi_{n}-\phi_{n+1}\right\|_{E_{0}}$, then

$$
\left\|\phi_{n}-\phi_{n+1}\right\|_{E_{0}} \leq \psi\left(\left\|\phi_{n}-\phi_{n+1}\right\|_{E_{0}}\right)<\left\|\phi_{n}-\phi_{n+1}\right\|_{E_{0}}
$$

which leads to a contradiction. Therefore, for each $n \in \mathbb{N}$, we have

$$
\left\|\phi_{n}-\phi_{n+1}\right\|_{E_{0}} \leq \psi\left(\left\|\phi_{n}-\phi_{n-1}\right\|_{E_{0}}\right)
$$

By induction, we obtain

$$
\left\|\phi_{n}-\phi_{n+1}\right\|_{E_{0}} \leq \psi^{n}\left(\left\|\phi_{0}-\phi_{1}\right\|_{E_{0}}\right)
$$

Fix $\varepsilon>0$. This implies that there exists $N \in \mathbb{N}$ such that

$$
\sum_{n \geq N} \psi^{n}\left(\left\|\phi_{0}-\phi_{1}\right\|_{E_{0}}\right)<\varepsilon
$$

For each $m, n \in \mathbb{N}$ with $m>n>N$, we obtain

$$
\left\|\phi_{n}-\phi_{m}\right\|_{E_{0}} \leq \sum_{k=n}^{m-1}\left\|\phi_{k}-\phi_{k+1}\right\|_{E_{0}} \leq \sum_{k=n}^{m-1} \psi^{k}\left(\left\|\phi_{0}-\phi_{1}\right\|_{E_{0}}\right) \leq \sum_{n \geq N} \psi^{n}\left(\left\|\phi_{0}-\phi_{1}\right\|_{E_{0}}\right)<\varepsilon .
$$

This implies that $\left\{\phi_{n}\right\}$ is a Cauchy sequence. By the completeness of $E_{0}$, we have $\lim _{n \rightarrow \infty} \phi_{n}=\phi$ for some $\phi \in E_{0}$ and

$$
\lim _{n \rightarrow \infty} T \phi_{n}=\lim _{n \rightarrow \infty} \phi_{n+1}(c)=\phi(c) .
$$

Since $\mathcal{R}_{c}$ is algebraically closed with respect to the norm topology, we have $\phi \in \mathcal{R}_{c}$. We next prove that $\phi$ is a PPF dependent fixed point of $T$. Using the continuity of $T$, we obtain $\lim _{n \rightarrow \infty} T \phi_{n}=T \phi$. By the uniqueness of the limit, we have $T \phi=\phi(c)$.

\section{Remark 2.4}

(i) From the proof of Theorem 2.3, we assume that the Razumikhin class $\mathcal{R}_{c}$ is algebraically closed with respect to difference, that is, $\phi-\alpha \in \mathcal{R}_{c}$ for all $\phi, \alpha \in \mathcal{R}_{c}$, in order to construct the sequence $\left\{\phi_{n}\right\}$ satisfying

$$
\left\|\phi_{n}-\phi_{n+1}\right\|_{E_{0}}=\left\|\phi_{n}(c)-\phi_{n+1}(c)\right\|_{E} \quad \text { for all } n \in \mathbb{N} \cup\{0\}
$$

(ii) In the proof of Theorem 2.3, if we choose $\phi_{n} \in \mathcal{R}_{c}$ to be a constant mapping for each $n \in \mathbb{N} \cup\{0\}$, then

$$
\left\|\phi_{n}-\phi_{n+1}\right\|_{E_{0}}=\left\|\phi_{n}(c)-\phi_{n+1}(c)\right\|_{E} \quad \text { for all } n \in \mathbb{N} \cup\{0\}
$$

Therefore the algebraic closedness with respect to the difference of $\mathcal{R}_{c}$ can be dropped. 
Example 2.5 Let $E=\mathbb{R}^{2}$ with respect to the norm $\|(x, y)\|=|x|+|y|$ and $E_{0}=C\left([0,1], \mathbb{R}^{2}\right)$. Define a mapping $\psi:[0, \infty) \rightarrow[0, \infty)$ by

$$
\psi(t)=\frac{t}{3} \quad \text { for all } t \in[0, \infty) \text {. }
$$

We see that $\psi$ is a nondecreasing mapping with $\sum_{n=1}^{\infty} \psi^{n}(t)<\infty$ for all $t \in(0,+\infty)$. Let $\phi \in E_{0}$. Therefore, for each $t \in[0,1]$, we obtain $\phi(t) \in \mathbb{R}^{2}$. Thus we can define mappings $f_{\phi}, g_{\phi}:[0,1] \rightarrow \mathbb{R}$ such that

$$
\phi(t)=\left(f_{\phi}(t), g_{\phi}(t)\right) \quad \text { for all } t \in[0,1]
$$

Define a mapping $T: E_{0} \rightarrow E$ by

$$
T \phi=\left(\frac{1}{4}\left\|f_{\phi}\right\|, \frac{1}{4}\left\|g_{\phi}\right\|\right) \quad \text { for all } \phi \in E_{0},
$$

where $\left\|f_{\phi}\right\|=\sup _{t \in[0,1]}\left|f_{\phi}(t)\right|$ and $\left\|g_{\phi}\right\|=\sup _{t \in[0,1]}\left|g_{\phi}(t)\right|$. Suppose that $\phi, \alpha \in E_{0}$ with $\phi \leq \alpha$. For each $c \in[0,1]$, we obtain

$$
\begin{aligned}
\|T \phi-T \alpha\|_{E} & =\left\|\left(\frac{1}{4}\left\|f_{\phi}\right\|, \frac{1}{4}\left\|g_{\phi}\right\|\right)-\left(\frac{1}{4}\left\|f_{\alpha}\right\|, \frac{1}{4}\left\|g_{\alpha}\right\|\right)\right\|_{E} \\
& =\left|\frac{1}{4}\left\|f_{\phi}\right\|-\frac{1}{4}\left\|f_{\alpha}\right\|\right|+\left|\frac{1}{4}\left\|g_{\phi}\right\|-\frac{1}{4}\left\|g_{\alpha}\right\|\right| \\
& \leq \frac{1}{4}\left(\left\|f_{\phi}-f_{\alpha}\right\|+\left\|g_{\phi}-g_{\alpha}\right\|\right) \\
& =\frac{1}{4}\left(\sup _{t \in[0,1]}\left|f_{\phi}(t)-f_{\alpha}(t)\right|+\sup _{t \in[0,1]}\left|g_{\phi}(t)-g_{\alpha}(t)\right|\right) \\
& =\frac{1}{4}\left(\sup _{t \in[0,1]}\left(\left|f_{\phi}(t)-f_{\alpha}(t)\right|+\left|g_{\phi}(t)-g_{\alpha}(t)\right|\right)\right) \\
& =\frac{1}{4}\left(\sup _{t \in[0,1]}\left\|\left(f_{\phi}(t)-f_{\alpha}(t), g_{\phi}(t)-g_{\alpha}(t)\right)\right\|_{E}\right) \\
& =\frac{1}{4}\left(\sup _{t \in[0,1]}\left\|\left(f_{\phi}(t), g_{\phi}(t)\right)-\left(f_{\alpha}(t), g_{\alpha}(t)\right)\right\|_{E}\right) \\
& =\frac{1}{4}\left(\sup _{t \in[0,1]}\|\phi(t)-\alpha(t)\|_{E}\right) \\
& =\frac{1}{4}\left(\|\phi-\alpha\|_{E_{0}}\right) \\
& \leq \psi\left(\operatorname { m a x } \left\{\|\phi-\alpha\|_{E_{0}},\|\phi(c)-T \phi\|_{E},\|\alpha(c)-T \alpha\|_{E}\right.\right. \\
& \left.\left.\frac{1}{2}\left[\|\phi(c)-T \alpha\|_{E}+\|\alpha(c)-T \phi\|_{E}\right]\right\}\right) .
\end{aligned}
$$

Suppose that $\phi_{0}=0$. We see that all assumptions in Theorem 2.3 are now satisfied and 0 is the PPF dependent fixed point of $T$ in $\mathcal{R}_{c}$.

By applying Theorem 2.3, we obtain the following corollary. 
Corollary 2.6 Suppose that $c \in I$ and $T: E_{0} \rightarrow E$ satisfies the following conditions:

(i) $T$ is a nondecreasing mapping;

(ii) for all $\phi, \alpha \in E_{0}$ with $\phi \leq \alpha$, we have

$$
\begin{aligned}
\|T \phi-T \alpha\|_{E} \leq & k\left(\operatorname { m a x } \left\{\|\phi-\alpha\|_{E_{0}},\|\phi(c)-T \phi\|_{E^{\prime}}\|\alpha(c)-T \alpha\|_{E}\right.\right. \\
& \left.\left.\frac{1}{2}\left[\|\phi(c)-T \alpha\|_{E}+\|\alpha(c)-T \phi\|_{E}\right]\right\}\right), \quad \text { where } k \in[0,1) ;
\end{aligned}
$$

(iii) there exists a lower solution $\phi_{0} \in \mathcal{R}_{c}$ such that $\phi_{0}(c) \leq T \phi_{0}$;

(iv) $T$ is a continuous mapping.

Assume that $\mathcal{R}_{c}$ is algebraically closed with respect to the difference. Then $T$ has a PPF dependent fixed point in $\mathcal{R}_{c}$.

Proof Define a function $\psi:[0,+\infty) \rightarrow[0,+\infty)$ by $\psi(t)=k t$ for all $t \in[0,+\infty)$. Therefore $\psi$ is a nondecreasing mapping and

$$
\sum_{n=1}^{\infty} \psi^{n}(t)<\infty \quad \text { for all } t \in(0,+\infty)
$$

This implies that all assumptions in Theorem 2.3 are satisfied. Hence we obtain the desired result.

Theorem 2.7 Suppose that $\psi \in \Psi_{1}, c \in I$ and $T: E_{0} \rightarrow$ Esatisfies the following conditions:

(i) $T$ is a nondecreasing mapping;

(ii) for all $\phi, \alpha \in E_{0}$ with $\phi \leq \alpha,\|T \phi-T \alpha\|_{E} \leq \psi\left(\|\phi-\alpha\|_{E_{0}}\right)$;

(iii) there exists a lower solution $\phi_{0} \in \mathcal{R}_{c}$ such that $\phi_{0}(c) \leq T \phi_{0}$;

(iv) if $\left\{\phi_{n}\right\}$ is a nondecreasing sequence in $E_{0}$ converging to $\phi \in E_{0}$, then $\phi_{n} \leq \phi$ for all $n \in \mathbb{N}$.

Assume that $\mathcal{R}_{c}$ is algebraically closed with respect to the difference. Then T has a PPF dependent fixed point in $\mathcal{R}_{c}$.

Proof By the analogous proof as in Theorem 2.3, we can construct a nondecreasing sequence $\left\{\phi_{n}\right\}$ in $\mathcal{R}_{c}$ converging to $\phi \in \mathcal{R}_{c}$. This implies that $\phi_{n} \leq \phi$ for all $n \in \mathbb{N}$. Therefore, for each $n \in \mathbb{N}$, we have

$$
\begin{aligned}
\|T \phi-\phi(c)\|_{E} & \leq\left\|T \phi-\phi_{n+1}(c)\right\|_{E}+\left\|\phi_{n+1}(c)-\phi(c)\right\|_{E} \\
& \leq\left\|T \phi-T \phi_{n}\right\|_{E}+\left\|\phi_{n+1}-\phi\right\|_{E_{0}} \\
& \leq \psi\left(\left\|\phi-\phi_{n}\right\|_{E_{0}}\right)+\left\|\phi_{n+1}-\phi\right\|_{E_{0}} .
\end{aligned}
$$

Since $\psi$ is continuous at 0 , we get $\lim _{n \rightarrow \infty} \psi\left(\left\|\phi-\phi_{n}\right\|_{E_{0}}\right)=\psi(0)=0$. Taking the limit of the above inequality, this yields $\|T \phi-\phi(c)\|_{E}=0$ and so $\phi$ is a PPF dependent fixed point of $T$ in $\mathcal{R}_{c}$.

We next ensure the result on PPF dependent fixed points for mappings concerning with $\psi \in \Psi_{2}$. 
Theorem 2.8 Suppose that $\psi \in \Psi_{2}, c \in I$ and $T: E_{0} \rightarrow$ E satisfies the following conditions:

(i) $T$ is a nondecreasing mapping;

(ii) for all $\phi, \alpha \in E_{0}$ with $\phi \leq \alpha$, we have

$$
\begin{aligned}
\|T \phi-T \alpha\|_{E} \leq & \psi\left(\operatorname { m a x } \left\{\|\phi-\alpha\|_{E_{0}},\|\phi(c)-T \phi\|_{E},\|\alpha(c)-T \alpha\|_{E},\right.\right. \\
& \left.\left.\frac{1}{2}\left[\|\phi(c)-T \alpha\|_{E}+\|\alpha(c)-T \phi\|_{E}\right]\right\}\right)
\end{aligned}
$$

(iii) there exists a lower solution $\phi_{0} \in \mathcal{R}_{c}$ such that $\phi_{0}(c) \leq T \phi_{0}$;

(iv) $T$ is a continuous mapping.

Assume that $\mathcal{R}_{c}$ is algebraically closed with respect to the difference. Then $T$ has a PPF dependent fixed point in $\mathcal{R}_{c}$.

Proof Since $\phi_{0} \in \mathcal{R}_{c}$ and $T \phi_{0} \in E$, there exists $x_{1} \in E$ such that $T \phi_{0}=x_{1}$. As in the proof of Theorem 2.3, we can construct the sequence $\left\{\phi_{n}\right\}$ such that

$$
\begin{aligned}
& T \phi_{0}=\phi_{1}(c) \leq T \phi_{1}=\phi_{2}(c) \leq \cdots \leq T \phi_{n}=\phi_{n+1}(c) \leq T \phi_{n+1} \cdots \\
& \phi_{n} \leq \phi_{n+1} \quad \text { and } \quad\left\|\phi_{n}-\phi_{n+1}\right\|_{E_{0}}=\left\|\phi_{n}(c)-\phi_{n+1}(c)\right\|_{E^{\prime}}
\end{aligned}
$$

for all $n \in \mathbb{N} \cup\{0\}$. Assume that $\phi_{n-1}=\phi_{n}$ for some $n \in \mathbb{N}$. It follows that $\phi_{n-1}(c)=\phi_{n}(c)=$ $T \phi_{n-1}$. Therefore $T$ has a fixed point in $\mathcal{R}_{c}$. Suppose that $\phi_{n-1} \neq \phi_{n}$ for all $n \in \mathbb{N}$. For each $n \in \mathbb{N}$, we obtain

$$
\begin{aligned}
\left\|\phi_{n}-\phi_{n+1}\right\|_{E_{0}}= & \left\|\phi_{n}(c)-\phi_{n+1}(c)\right\|_{E} \\
= & \left\|T \phi_{n}-T \phi_{n-1}\right\|_{E} \\
\leq & \psi\left(\operatorname { m a x } \left\{\left\|\phi_{n}-\phi_{n-1}\right\|_{E_{0}},\left\|\phi_{n}(c)-T \phi_{n}\right\|_{E},\left\|\phi_{n-1}(c)-T \phi_{n-1}\right\|_{E},\right.\right. \\
& \left.\left.\frac{1}{2}\left[\left\|\phi_{n}(c)-T \phi_{n-1}\right\|_{E}+\left\|\phi_{n-1}(c)-T \phi_{n}\right\|_{E}\right]\right\}\right) \\
= & \psi\left(\operatorname { m a x } \left\{\left\|\phi_{n}-\phi_{n-1}\right\|_{E_{0}},\left\|\phi_{n}(c)-\phi_{n+1}(c)\right\|_{E},\left\|\phi_{n-1}(c)-\phi_{n}(c)\right\|_{E^{\prime}}\right.\right. \\
& \left.\left.\frac{1}{2}\left[\left\|\phi_{n}(c)-\phi_{n}(c)\right\|_{E}+\left\|\phi_{n-1}(c)-\phi_{n+1}(c)\right\|_{E}\right]\right\}\right) \\
\leq & \psi\left(\max \left\{\left\|\phi_{n}-\phi_{n-1}\right\|_{E_{0}},\left\|\phi_{n}-\phi_{n+1}\right\|_{E_{0}}, \frac{1}{2}\left\|\phi_{n-1}-\phi_{n+1}\right\|_{E_{0}}\right\}\right) \\
\leq & \psi\left(\operatorname { m a x } \left\{\left\|\phi_{n}-\phi_{n-1}\right\|_{E_{0}},\left\|\phi_{n}-\phi_{n+1}\right\|_{E_{0}},\right.\right. \\
& \left.\left.\frac{1}{2}\left\|\phi_{n-1}-\phi_{n}\right\|_{E_{0}}+\left\|\phi_{n}-\phi_{n+1}\right\|_{E_{0}}\right\}\right) \\
\leq & \psi\left(\max \left\{\left\|\phi_{n}-\phi_{n-1}\right\|_{E_{0}},\left\|\phi_{n}-\phi_{n+1}\right\|_{E_{0}}\right\}\right) .
\end{aligned}
$$


If $\max \left\{\left\|\phi_{n}-\phi_{n-1}\right\|_{E_{0}},\left\|\phi_{n}-\phi_{n+1}\right\|_{E_{0}}\right\}=\left\|\phi_{n}-\phi_{n+1}\right\|_{E_{0}}$, then

$$
\left\|\phi_{n}-\phi_{n+1}\right\|_{E_{0}} \leq \psi\left(\left\|\phi_{n}-\phi_{n+1}\right\|_{E_{0}}\right)<\left\|\phi_{n}-\phi_{n+1}\right\|_{E_{0}}
$$

This leads to a contradiction. Therefore

$$
\begin{aligned}
\left\|\phi_{n}-\phi_{n+1}\right\|_{E_{0}} & \leq \psi\left(\left\|\phi_{n}-\phi_{n-1}\right\|_{E_{0}}\right) \\
& <\left\|\phi_{n}-\phi_{n-1}\right\|_{E_{0}} .
\end{aligned}
$$

It follows that $\left\|\phi_{n}-\phi_{n+1}\right\|_{E_{0}} \leq\left\|\phi_{n-1}-\phi_{n}\right\|_{E_{0}}$ for all $n \in \mathbb{N}$. Since the sequence $\left\{\| \phi_{n}-\right.$ $\left.\phi_{n+1} \|_{E_{0}}\right\}$ is a nonincreasing sequence of nonnegative real numbers, we see that it is a convergent sequence. Suppose that

$$
\lim _{n \rightarrow \infty}\left\|\phi_{n}-\phi_{n+1}\right\|_{E_{0}}=\alpha
$$

for some nonnegative real number $\alpha$. We will prove that $\alpha=0$. Suppose that $\alpha>0$. Since

$$
\left\|\phi_{n}-\phi_{n+1}\right\|_{E_{0}} \leq \psi\left(\left\|\phi_{n}-\phi_{n-1}\right\|_{E_{0}}\right)
$$

for all $n \in \mathbb{N}$ and the continuity of $\psi$, we have $\alpha \leq \psi(\alpha)<\alpha$ which leads to a contradiction. This implies that $\alpha=0$. We next prove that the sequence $\left\{\phi_{n}\right\}$ is a Cauchy sequence in $E_{0}$. Assume that $\left\{\phi_{n}\right\}$ is not a Cauchy sequence. It follows that there exist $\varepsilon>0$ and two sequences of positive integers $\left\{m_{k}\right\}$ and $\left\{n_{k}\right\}$ satisfying $m_{k}>n_{k}>k$ for each $k \in \mathbb{N}$ and

$$
\left\|\phi_{m_{k}}-\phi_{n_{k}}\right\|_{E_{0}} \geq \varepsilon
$$

Let $\left\{m_{k}\right\}$ be the sequence of the least positive integers exceeding $\left\{n_{k}\right\}$ which satisfies (2.1) and

$$
\left\|\phi_{m_{k}-1}-\phi_{n_{k}}\right\|_{E_{0}}<\varepsilon
$$

We will prove that $\lim _{k \rightarrow \infty}\left\|\phi_{m_{k}}-\phi_{n_{k}}\right\|_{E_{0}}=\varepsilon$. Since $\left\|\phi_{m_{k}}-\phi_{n_{k}}\right\|_{E_{0}} \geq \varepsilon$ for all $k \in \mathbb{N}$, we have

$$
\lim _{k \rightarrow \infty}\left\|\phi_{m_{k}}-\phi_{n_{k}}\right\|_{E_{0}} \geq \varepsilon
$$

For each $k \in \mathbb{N}$, we obtain

$$
\begin{aligned}
\left\|\phi_{m_{k}}-\phi_{n_{k}}\right\|_{E_{0}} & \leq\left\|\phi_{m_{k}}-\phi_{m_{k}-1}\right\|_{E_{0}}+\left\|\phi_{m_{k}-1}-\phi_{n_{k}}\right\|_{E_{0}} \\
& \leq\left\|\phi_{m_{k}}-\phi_{m_{k}-1}\right\|_{E_{0}}+\varepsilon .
\end{aligned}
$$

This implies that $\lim _{k \rightarrow \infty}\left\|\phi_{m_{k}}-\phi_{n_{k}}\right\|_{E_{0}} \leq \varepsilon$. Therefore

$$
\lim _{k \rightarrow \infty}\left\|\phi_{m_{k}}-\phi_{n_{k}}\right\|_{E_{0}}=\varepsilon
$$


Similarly, we can prove that

$$
\lim _{k \rightarrow \infty}\left\|\phi_{m_{k}+1}-\phi_{n_{k}}\right\|_{E_{0}}=\varepsilon, \quad \lim _{k \rightarrow \infty}\left\|\phi_{m_{k}}-\phi_{n_{k}-1}\right\|_{E_{0}}=\varepsilon
$$

and

$$
\lim _{k \rightarrow \infty}\left\|\phi_{m_{k}+1}-\phi_{n_{k}-1}\right\|_{E_{0}}=\varepsilon
$$

Since $\mathcal{R}_{c}$ is algebraically closed with respect to the difference, for each $k \in \mathbb{N}$, we obtain

$$
\begin{aligned}
\left\|\phi_{n_{k}}-\phi_{m_{k}+1}\right\|_{E_{0}}= & \left\|\phi_{n_{k}}(c)-\phi_{m_{k}+1}(c)\right\|_{E} \\
= & \left\|T \phi_{m_{k}}-T \phi_{n_{k}-1}\right\|_{E} \\
\leq & \psi\left(\operatorname { m a x } \left\{\left\|\phi_{m_{k}}-\phi_{n_{k}-1}\right\|_{E_{0}},\left\|\phi_{m_{k}}(c)-T \phi_{m_{k}}\right\|_{E^{\prime}}\right.\right. \\
& \left\|\phi_{n_{k}-1}(c)-T \phi_{n_{k}-1}\right\|_{E}, \\
& \left.\left.\frac{1}{2}\left[\left\|\phi_{m_{k}}(c)-T \phi_{n_{k}-1}\right\|_{E}+\left\|\phi_{n_{k}-1}(c)-T \phi_{n_{k}}\right\|_{E}\right]\right\}\right) \\
= & \psi\left(\operatorname { m a x } \left\{\left\|\phi_{m_{k}}-\phi_{n_{k}-1}\right\|_{E_{0}},\left\|\phi_{m_{k}}(c)-\phi_{m_{k}+1}(c)\right\|_{E^{\prime}}\right.\right. \\
& \left\|\phi_{n_{k}-1}(c)-\phi_{n_{k}}(c)\right\|_{E^{\prime}} \\
& \left.\left.\frac{1}{2}\left[\left\|\phi_{m_{k}}(c)-\phi_{n_{k}}(c)\right\|_{E}+\left\|\phi_{n_{k}-1}(c)-\phi_{n_{k}+1}(c)\right\|_{E}\right]\right\}\right) \\
\leq & \psi\left(\operatorname { m a x } \left\{\left\|\phi_{m_{k}}-\phi_{n_{k}-1}\right\|_{E_{0}},\left\|\phi_{m_{k}}-\phi_{m_{k}+1}\right\|_{E_{0}},\left\|\phi_{n_{k}-1}-\phi_{n_{k}}\right\|_{E_{0}}\right.\right. \\
& \left.\left.\frac{1}{2}\left[\left\|\phi_{m_{k}}-\phi_{n_{k}}\right\|_{E_{0}}+\left\|\phi_{n_{k}-1}-\phi_{n_{k}+1}\right\|_{E_{0}}\right]\right\}\right) \\
\leq & \psi\left(\operatorname { m a x } \left\{\left\|\phi_{m_{k}}-\phi_{n_{k}-1}\right\|_{E_{0}},\left\|\phi_{m_{k}}-\phi_{m_{k}+1}\right\|_{E_{0}},\left\|\phi_{n_{k}-1}-\phi_{n_{k}}\right\|_{E_{0}},\right.\right. \\
& \left.\left.\frac{1}{2}\left[\left\|\phi_{m_{k}}-\phi_{n_{k}}\right\|_{E_{0}}+\left\|\phi_{n_{k}-1}-\phi_{n_{k}}\right\|_{E_{0}}+\left\|\phi_{n_{k}}-\phi_{n_{k}+1}\right\|_{E_{0}}\right]\right\}\right) \\
& \\
& \\
&
\end{aligned}
$$

By taking the limit of both sides, we have

$$
\varepsilon \leq \psi(\varepsilon)<\varepsilon
$$

This leads to a contradiction. It follows that the sequence $\left\{\phi_{n}\right\}$ is a Cauchy sequence. By the completeness of $E_{0}$, we have $\lim _{n \rightarrow \infty} \phi_{n}=\phi$ for some $\phi \in E_{0}$ and

$$
\lim _{n \rightarrow \infty} T \phi_{n}=\lim _{n \rightarrow \infty} \phi_{n+1}(c)=\phi(c) .
$$

Since $\mathcal{R}_{c}$ is algebraically closed with respect to the norm topology, we have $\phi \in \mathcal{R}_{c}$. We will prove that $\phi$ is a PPF dependent fixed point of $T$. Using the continuity of $T$, we obtain $\lim _{n \rightarrow \infty} T \phi_{n}=T \phi$. By the uniqueness of the limit, we can conclude that $T \phi=\phi(c)$. 
Example 2.9 Assume that $E=\mathbb{R}$ and $E_{0}=C([0,1], \mathbb{R})$. Define a mapping $\psi:[0, \infty) \rightarrow$ $[0, \infty)$ by

$$
\psi(t)=\frac{t}{2} \quad \text { for all } t \in[0, \infty)
$$

We see that $\psi$ is a continuous nondecreasing mapping with $\psi(t)<t$. Define a mapping $T: E_{0} \rightarrow E$ by

$$
T \phi=\frac{1}{3} \phi\left(\frac{1}{4}\right) \text { for all } \phi \in E_{0} \text {. }
$$

Suppose that $\phi, \alpha \in E_{0}$ with $\psi \leq \alpha$ and $c \in[0,1]$. Therefore

$$
\begin{aligned}
\|T \phi-T \alpha\|_{E}= & \frac{1}{3}\left|\phi\left(\frac{1}{4}\right)-\alpha\left(\frac{1}{4}\right)\right| \\
\leq & \frac{1}{3}\|\phi-\alpha\|_{E_{0}} \\
\leq & \psi\left(\operatorname { m a x } \left\{\|\phi-\alpha\|_{E_{0}},\|\phi(c)-T \phi\|_{E},\|\alpha(c)-T \alpha\|_{E}\right.\right. \\
& \left.\left.\frac{1}{2}\left[\|\phi(c)-T \alpha\|_{E}+\|\alpha(c)-T \phi\|_{E}\right]\right\}\right) .
\end{aligned}
$$

Suppose that $\phi_{0}=0$. We find that all assumptions in Theorem 2.8 are now satisfied and 0 is the PPF dependent fixed point of $T$ in $\mathcal{R}_{c}$.

For the next result, we drop the continuity of $T$.

Theorem 2.10 Suppose that $\psi \in \Psi_{2}, c \in I$, and that $T: E_{0} \rightarrow E$ satisfies the following conditions:

(i) $T$ is a nondecreasing mapping;

(ii) for all $\phi, \alpha \in E_{0}$ with $\phi \leq \alpha$, we have

$$
\begin{aligned}
\|T \phi-T \alpha\|_{E} \leq & \psi\left(\operatorname { m a x } \left\{\|\phi-\alpha\|_{E_{0}},\|\phi(c)-T \phi\|_{E^{\prime}}\|\alpha(c)-T \alpha\|_{E^{\prime}}\right.\right. \\
& \left.\left.\frac{1}{2}\left[\|\phi(c)-T \alpha\|_{E}+\|\alpha(c)-T \phi\|_{E}\right]\right\}\right) ;
\end{aligned}
$$

(iii) there exists a lower solution $\phi_{0} \in \mathcal{R}_{c}$ such that $\phi_{0}(c) \leq T \phi_{0}$;

(iv) if $\left\{\phi_{n}\right\}$ is a nondecreasing sequence in $E_{0}$ converging to $\phi \in E_{0}$, then $\phi_{n} \leq \phi$ for all $n \in \mathbb{N}$.

Assume that $\mathcal{R}_{c}$ is algebraically closed with respect to the difference. Then $T$ has a PPF dependent fixed point in $\mathcal{R}_{c}$.

Proof As in the proof of Theorem 2.8, we can construct a nondecreasing sequence $\left\{\phi_{n}\right\}$ converging to $\phi \in \mathcal{R}_{c}$ and this yields

$$
\lim _{n \rightarrow \infty} T \phi_{n}=\lim _{n \rightarrow \infty} \phi_{n+1}(c)=\phi(c) .
$$


Using (iv), we have $\phi_{n} \leq \phi$ for all $n \in \mathbb{N}$. Therefore, for each $n \in \mathbb{N}$, we obtain

$$
\begin{aligned}
\|T \phi-\phi(c)\|_{E} \leq & \left\|T \phi-\phi_{n+1}(c)\right\|_{E}+\left\|\phi_{n+1}(c)-\phi(c)\right\|_{E} \\
\leq & \left\|T \phi-T \phi_{n}\right\|_{E}+\left\|\phi_{n+1}-\phi\right\|_{E_{0}} \\
\leq & \psi\left(\operatorname { m a x } \left\{\left\|\phi-\phi_{n}\right\|_{E_{0}},\|\phi(c)-T \phi\|_{E},\left\|\phi_{n}(c)-T \phi_{n}\right\|_{E},\right.\right. \\
& \left.\left.\frac{1}{2}\left[\left\|\phi(c)-T \phi_{n}\right\|_{E}+\left\|\phi_{n}(c)-T \phi\right\|_{E}\right]\right\}\right)+\left\|\phi_{n+1}-\phi\right\|_{E_{0}} \\
= & \psi\left(\operatorname { m a x } \left\{\left\|\phi-\phi_{n}\right\|_{E_{0}},\|\phi(c)-T \phi\|_{E},\left\|\phi_{n}(c)-\phi_{n+1}(c)\right\|_{E},\right.\right. \\
& \left.\left.\frac{1}{2}\left[\left\|\phi(c)-\phi_{n+1}(c)\right\|_{E}+\left\|\phi_{n}(c)-T \phi\right\|_{E}\right]\right\}\right)+\left\|\phi_{n+1}-\phi\right\|_{E_{0}} \\
\leq & \psi\left(\operatorname { m a x } \left\{\left\|\phi-\phi_{n}\right\|_{E_{0}},\|\phi(c)-T \phi\|_{E},\left\|\phi_{n}-\phi_{n+1}\right\|_{E_{0}}\right.\right. \\
& \left.\left.\frac{1}{2}\left[\left\|\phi-\phi_{n+1}\right\|_{E_{0}}+\left\|\phi_{n}(c)-T \phi\right\|_{E}\right]\right\}\right)+\left\|\phi_{n+1}-\phi\right\|_{E_{0}} .
\end{aligned}
$$

Letting $n \rightarrow \infty$, we obtain $\|T \phi-\phi(c)\|_{E} \leq \psi\left(\|\phi(c)-T \phi\|_{E}\right)$. If $\phi(c) \neq T \phi$, then

$$
\|T \phi-\phi(c)\|_{E} \leq \psi\left(\|\phi(c)-T \phi\|_{E}\right)<\|\phi(c)-T \phi\|_{E}
$$

This leads to a contradiction. Therefore $T \phi=\phi(c)$. This implies that $\phi$ is a PPF dependent fixed point of $T$.

By applying Theorem 2.8 and Theorem 2.10, we obtain the following corollary.

Corollary 2.11 Suppose that $c \in I$ and that $T: E_{0} \rightarrow$ E satisfies the following conditions:

(i) $T$ is a nondecreasing mapping;

(ii) for all $\phi, \alpha \in E_{0}$ with $\phi \leq \alpha$, we have

$$
\begin{aligned}
\|T \phi-T \alpha\|_{E} \leq & k\left(\operatorname { m a x } \left\{\|\phi-\alpha\|_{E_{0}},\|\phi(c)-T \phi\|_{E},\|\alpha(c)-T \alpha\|_{E},\right.\right. \\
& \left.\left.\frac{1}{2}\left[\|\phi(c)-T \alpha\|_{E}+\|\alpha(c)-T \phi\|_{E}\right]\right\}\right), \quad \text { where } k \in[0,1)
\end{aligned}
$$

(iii) there exists a lower solution $\phi_{0} \in \mathcal{R}_{c}$ such that $\phi_{0}(c) \leq T \phi_{0}$;

(iv) $T$ is a continuous mapping or if $\left\{\phi_{n}\right\}$ is a nondecreasing sequence in $E_{0}$ converging to $\phi \in E_{0}$, then $\phi_{n} \leq \phi$ for all $n \in \mathbb{N}$.

Assume that $\mathcal{R}_{c}$ is algebraically closed with respect to the difference. Then $T$ has a PPF dependent fixed point in $\mathcal{R}_{c}$.

Proof Define a function $\psi:[0,+\infty) \rightarrow[0,+\infty)$ by $\psi(t)=k t$ for all $t \in[0,+\infty)$. Therefore $\psi$ is a continuous nondecreasing mapping and

$$
\psi(t)<t \quad \text { for all } t \in(0,+\infty) \text {. }
$$


This implies that all assumptions in Theorem 2.8 or Theorem 2.10 are satisfied. Hence the proof is complete.

\section{Competing interests}

The authors declare that they have no competing interests.

\section{Authors' contributions}

All authors contributed equally to the writing of this paper. All authors read and approved the final manuscript.

\section{Author details}

${ }^{1}$ Department of Mathematics, Faculty of Science, Razi University, Kermanshah, 67149, Iran. ${ }^{2}$ Department of Mathematics, Faculty of Science, Naresuan University, Phitsanulok, 65000, Thailand. ${ }^{3}$ Centre of Excellence in Mathematics, CHE, Si Ayutthaya Rd., Bangkok, 10400, Thailand.

\section{Acknowledgements}

The second author would like to express her deep thanks to the Centre of Excellence in Mathematics, the Commission of Higher Education and Naresuan University, Thailand for the support.

Received: 10 October 2013 Accepted: 10 September 2014 Published: 26 Sep 2014

\section{References}

1. Beg, l, Abbas, M: Coincidence point and invariant approximation for mappings satisfying generalized weak contractive condition. Fixed Point Theory Appl. (2006). doi:10.1155/2006/74503

2. Drici, Z, McRae, FA, Vasundhara Devi, J: Fixed point theorem in partially ordered metric spaces for operators with PPF dependence. Nonlinear Anal. 67, 641-647 (2007)

3. Jungck, G: Compatible mappings and common fixed points. Int. J. Math. Sci. 9, 771-779 (1968)

4. Jungck, G: Common fixed points for commuting and compatible maps on compacta. Proc. Am. Math. Soc. 103 977-983 (1988)

5. Jungck, G: Common fixed points for noncontinuous nonself maps on nonmetric spaces. Far East J. Math. Sci. 4, 199-215 (1996)

6. Jungck, G, Hussain, N: Compatible maps and invariant approximations. J. Math. Model. Algorithms 325, $1003-1012$ (2007)

7. Kaewcharoen, A: PPF dependent common fixed point theorems for mappings in Banach spaces. J. Inequal. Appl. (2013). doi:10.1186/1029-242X-2013-287

8. Pant, RP: Common fixed points of noncommuting mappings. J. Math. Anal. Appl. 188, 436-440 (1994)

9. Sintunavarat, W, Kumam, P: Common fixed point theorems for generalized $\mathcal{J} \mathcal{H}$-operators classed and invariant approximations. J. Inequal. Appl. (2011). doi:10.1186/1029-242X-2011-67

10. Bernfeld, SR, Lakshmikatham, V, Reddy, YM: Fixed point theorems of operators with PPF dependence in Banach spaces. Appl. Anal. 6, 271-280 (1977)

11. Dhage, BC: On some common fixed point theorems with PPF dependence in Banach spaces. J. Nonlinear Sci. Appl. 5 220-232 (2012)

12. Samet, B, Vetro, C, Vetro, P: Fixed point theorems for $\alpha$ - $\psi$-contractive type mappings. Nonlinear Anal. 75, 2154-2165 (2012)

\section{Submit your manuscript to a SpringerOpen ${ }^{\circ}$ journal and benefit from:}

- Convenient online submission

Rigorous peer review

- Immediate publication on acceptance

- Open access: articles freely available online

- High visibility within the field

- Retaining the copyright to your article 